\section{Elektronischer Heilberufeausweis: Alternative zum Ident-Verfahren}

Um von der Förderung der elektronischen Arztbriefe in 2017 profitieren zu können, benötigen Ärzte einen elektronischen Heilberufeausweis. Eigentlich müssen sie den Antrag dafür persönlich bei einer zugelassenen Identifizierungsstelle vorlegen und sich vor Ort ausweisen. Kunden der Deutschen Apotheker- und Ärztebank (apoBank) können sich diesen Weg sparen und den Ausweis direkt über die Bank online beantragen (www.apobank.de/earztausweis). Ihre Identität werde durch den Abgleich der bei der Bank bereits hinterlegten Daten geprüft, heißt es. Daher genüge es, wenn der Antrag online ausgefüllt, ausgedruckt und unterschrieben direkt an die zuständige Kammer gesendet wird.

Rebekka Höh

\section{DGN für qualifizierten \\ Zeitstempeldienst zertifiziert}

Seit 1. Juli 2016 ist eine neue EU-Signaturverordnung, die eIDAS (electronic Identification and Signature) in Kraft. Das Unternehmen DGN Deutsches Gesundheitsnetz bekam nun als erstes den Status "qualifizierter Vertrauensdiensteanbieter" für elektronische Zeitstempel gemäß eIDAS verliehen und zwar von der Bundesnetzagentur. Qualifizierte elektronische Zeitstempel ermöglichen einen vertrauenswürdigen Nachweis über den Erfassungszeitpunkt und Inhalt digitaler Dokumente. So lässt sich etwa in elektronischen Archiven rechtsverbindlich nachweisen, dass Signaturen zu einem bestimmten Zeitpunkt erfolgt sind. red

\section{D2D: Für den Umstieg rüsten}

Am 30. September 2016 wird die Telematik-Plattform D2D (Doctor-to-Doctor) abgeschaltet. Das betrifft bundesweit rund 13.000 Ärzte, die nicht nur elektronische Arztbriefe über die sichere Plattform verschicken. Nach Wunsch der Telematik $\mathrm{GmbH}$, die die Plattform betreibt, sollen die Ärzte künftig den moderneren Kommunikationskanal „KV-Connect“ nutzen und informiert Ärzte daher auf ihrer Website (www.kv-telematik.de), was diese bei der Umstellung beachten müssen. Voraussetzung für die Teilnahme an KVConnect ist etwa, dass sich die Ärzte um einen Zugang zum "Sicheren Netz der KVen“ (SNK) kümmern. RebekkaHöhl kalender kostenfrei an. Finanzieren will Gründer Stefan Knobl die Software, die dahintersteht, über Werbepartner. „Wir wollen aber keine Patientendaten auslesen“, sagt er. „Wir schauen einfach, bei welchen Fachrichtungen und in welchen Regionen werden Arzttermine gebucht." Dabei setze man dann auf regionale Werbepartner, etwa Sanitätshäuser vor Ort, zu denen Anzeigen eingespielt werden. Der Terminkalender soll vorrangig auf der Praxiswebsite eingebunden werden. Ärzte, die keine eigene Website haben, können den Terminkalender aber auch auf der Plattform facharzt-sofort. de einrichten - ebenfalls kostenfrei. Hier könnten sie dann zusätzlich ein Profil der Praxis mit Logo und Fotos hinterlegen, erläutert Knobl. Für die Verknüpfung mit dem eigenen Outlook- oder Google-Kalender des Arztes gibt es bereits eine Standardschnittstelle. Das Unternehmen arbeite aber auch daran, sich mit Praxissoftware-Anbietern zusammen zu tun und eine Direktanbindung in das Praxisverwaltungssystem (PVS) zu ermöglichen. So gibt es etwa schon eine Schnittstelle zur Software Medical Office des Anbieters Indamed, über die die Termine im Online-Kalender und im Kalender der Praxissoftware in Echtzeit abgeglichen werden.

Auch bei Facharzt-Sofort müssen sich die Patienten zunächst mit ihrer E-Mail registrieren. Anschließend erhalten sie einen Link, den sie bestätigen müssen. „Jeder Patient kann nur fünf Terminanfragen gleichzeitig laufen lassen", so Knobl. IP-Adressen - also im Prinzip die Rechnerkennung im Internet - mit falscher Identifizierung würden zudem verbannt. Alle Daten werden laut Knobl verschlüsselt übertragen, die Server stehen in Deutschland. Spannend ist, dass das Start-up aus Bayern auch eine $\mathrm{Zu}$ weiserplattform anbietet, in der sich Arztpraxen verschiedener Fachgruppen für die schnellere Terminvergabe untereinander vernetzen können. „Fast so wie über Facebook“, sagt Knobl.

\section{Serviceangebote oben drauf}

So ganz neu sind viele der Features der beiden Newcomer allerdings nicht. Die Integration in die Praxis-EDV, über die das Online-Terminsystem dann direkt gesteuert wird, ist bei samedi etwa prin- zipiell in der Comfort-Version enthalten. Dabei integrieren die Praxen laut samedi-Geschäftsführer Professor Alexander Alscher die Online-Buchungsmöglichkeit direkt in ihre Website. Außerdem könnten sie ihren Patienten eine App oder das Online-Patientenkonto über samedi.de zur Verfügung stellen. Das Patientenkonto biete dabei zusätzliche Serviceangebote für Patienten, wie eine sichere Online-Kommunikation mit dem Arzt oder das Stornieren von Terminen via Web. Gleichzeitig biete es aber auch einen höheren Schutz vor Terminausfällen, weil die Patienten eben ihre Buchungen online verfolgen können. Alscher sieht samedi auch mehr als Praxismanagementlösung. Denn neben dem Festlegen verschiedener Terminarten könnte die Praxis steuern, nach welchen Regeln sie ausgelastet werden solle nach Terminartgruppen, Terminketten, Teilterminen, Ressourcen mit austauschbaren Fähigkeiten oder dynamischen Verfallszeiten. Die samedi-Technik steckt beispielsweise auch im OnlineTerminkalender x.time, der für die Praxisverwaltungssysteme der medatixx dazu gebucht werden kann.

\section{Schnittstelle zur Praxissoftware}

Und selbst die Kombination Arztsuche und Online-Terminkalender ist keine komplette Neuheit: Das Arztbewertungsportal jameda.de bietet Ärzten schon länger ein kostenfreies OnlineTerminsystem, das sowohl über das Bewertungsportal als auch über einen Programmier-Code (HTML-Code) für die Einbindung in die eigene Praxiswebsite zur Verfügung steht, wie Unternehmenssprecherin Elke Ruppert erläutert. Dabei könnten die Ärzte ebenfalls definieren, für welche Leistungen Patienten bestimmte Termine buchen können, welche Behandler für welche Leistung zur Verfügung stehen und wie lange bestimmte Termine dauern. Allerdings läuft auch hier der Online-Kalender zunächst neben der Praxis-EDV. „Parallel dazu bieten wir für die Praxen, die ihre Termine weiter im gewohnten Praxiskalender bearbeiten möchten, auch technische Schnittstellen an“, so Ruppert. Terminerinnerungen per E-Mail und/oder SMS bieten dabei übrigens alle der etablierten Systeme.

RebekkaHöh 